\title{
Fditorial
}

\section{Benefits of smoking cessation for coronary heart disease patients}

\author{
Adi Hidayat \\ Department of Community Medicine, Faculty of Medicine, Trisakti University
}

Cardiovascular disease (CVD) incidence increases with age and is frequently higher in the elderly. ${ }^{(1)}$ Therefore prevention of CVD in the elderly through management of risk factors is important in order to reduce the risk of coronary heart disease (CHD). There are several risk factors of CVD that can be modified, such as smoking, physical activity, and unhealthy diet. Cessation of smoking is the most potent measure to prevent thousands of CVD events and deaths. (2)

Smoking is a modifiable major risk factor that causes several types of disease resulting in death. Various epidemiological studies in the last five decades have demonstrated a causal relationship between smoking on the one hand and CHD and mortality on the other. ${ }^{(3)}$

During these decades mortality from CVD had started to decrease in developed countries with the reduction in the number of smokers, but CVD is still the main cause of death.

One study found that cessation of smoking in the elderly is still of benefit. ${ }^{(4)}$ A metaanalytical study involving 503,905 subjects aged 60 years and older showed a significant association between smoking status and CVD mortality in smokers, with a hazard ratio of 2.07 (95\%CI: $1.82-2.36)$ for current smokers and 1.37 (95\% CI: 1.25-1.49) for former smokers in comparison with never-smokers. ${ }^{(5)}$ The risk in smokers increased with increasing cigarette consumption in a dose-response manner, and decreased continously with time in former smokers after they ceased to smoke. An European study on 1,439 subjects aged $\geq 40$ years who smoked $>10$ cigarettes per day showed a consistently high CV risk from smoking on the basis of the prediction models employed. According to the Systematic Coronary Risk Evaluation (SCORE) model, the probability of death from CVD in the next 10 years was twice that of non-smokers. ${ }^{(6)}$

Consistent study results showing increased risks relative to CVD occurrence and mortality have become more important for eliminating smoking as the source of these studies and CVD mortality, through smoking cessation programs. $^{(7)}$

Cessation of smoking as early as possible is associated with increased survival rate and quality of life, and reduces health care costs for smoking-related diseases. The principal benefit of smoking cessation lies in improved cardiovascular health. ${ }^{(8)}$ Communication on the risks of smoking and on the benefits of cessation of smoking is the most effective promotion effort. In order to eliminate the health and economic burden of CVD, future country-specific interventions on smoking cessation should be based on improved community-based smoking cessation programs at the primary health level. Such measures as counseling and pharmacotherapy can be used by primary health practitioners to influence the results of smoking 
cessation efforts. Simple advice from GPs has been shown to substantially increase smoking cessation rates. ${ }^{(9)}$

\section{REFERENCES}

1. Riekard P, Gharacholou SM, Peterson ED. Primary and secondary prevention of cardiovascular disease in older adults: a status report. Clin Geriatr Med 2009;25:745-55.

2. Aung MN, Yuasa M, Lorga T, et al. Evidencebased new service package vs. routine service package for smoking cessation to prevent high risk patients from cardiovascular diseases (CVD): study protocol for randomized controlled. Trials 2013;14:419-32.

3. US Department of Health and Human Services. The health consequences of smoking - 50 years of progress: a report of the Surgeon General. Atlanta, GA: US Department of Health and Human Services, Centers for Disease Control and Prevention, National Center for Chronic Disease Prevention and Health Promotion, Office on Smoking and Health, 2014.

4. Gellert C, Schottker B, Muller H, et al. Impact of smoking and quitting on cardiovascular outcomes and risk advancement periods among older adults. Eur J Epidemiol 2013;28:649-58.
5. Mons U, Muezzinler A, Gellert C, et al. Impact of smoking and smoking cessation on cardiovascular events and mortality among older adults: meta-analysis of individual participant data from prospective cohort studies of the CHANCES consortium. BMJ 2015;350:h1551. doi:10.1136/bmj.h1551.

6. Mallaina P, Lionis C, Ro H, et al. Smoking cessation and the risk of cardiovascular disease outcomes predicted from established risk scores: results of the cardiovascular risk assessment among smokers in primary care in Europe (CVASPIRE) study. BMC Public Health 2013,13: 362.

7. Gellert C, Schottker B, Muller H, et al. Impact of smoking and quitting on cardiovascular outcomes and risk advancement periods among older adults. Eur J Epidemiol 2013;28:649-58.

8. Mills EJ, Thorlund K, Eapen S, et al. Cardiovascular events associated with smoking cessation pharmacotherapies: a network metaanalysis. Circulation 2014;129:28-41. doi:10. 1161/CIRCULATIONAHA.113.003961.

9. Sadowski J, Ruffieux C, Cornuz J. Self-reported smoking cessation activities among Swiss primary care physicians. BMC Fam Pract 2009; 10:22. 